\title{
Erratum to: Safety, Formulation and In Vitro Antiviral Activity of the Antimicrobial Peptide Subtilosin Against Herpes Simplex Virus Type 1
}

\author{
Nicolás I. Torres $\cdot$ Katia Sutyak Noll $\cdot$ Shiqi Xu • \\ Ji Li • Qingrong Huang • Patrick J. Sinko • \\ Mónica B. Wachsman · Michael L. Chikindas
}

Published online: 16 February 2013

(C) Springer Science+Business Media New York 2013

\section{Erratum to: Probiotics \& Antimicro. Prot. DOI 10.1007/s12602-012-9123-x}

In the original version of this paper, the article note (Transparency declaration) was missed out unfortunately, which should have come in the front page of the article. The missed article note is as follows.
Transparency declaration: Nicolás I. Torres, Katia Sutyak Noll and Shiqi Xu equally contributed to the research and to this manuscript. Mónica B. Wachsman, Michael L. Chikindas and Qingrong Huang are the manuscript's senior authors in the areas of virology, bacteriology and formulation chemistry, correspondingly.

The online version of the original article can be found under doi: 10.1007/s12602-012-9123-x.

N. I. Torres - M. B. Wachsman

Laboratorio de Virología, Departamento de Química Biológica,

Facultad de Ciencias Exactas y Naturales, Universidad de

Buenos Aires, Ciudad Universitaria, Pabellón 2, Piso 4, 1428

Buenos Aires, Argentina

K. Sutyak Noll $\cdot$ S. Xu · J. Li · Q. Huang ·

M. L. Chikindas $(\square)$

School of Environmental and Biological Sciences,

Rutgers, The State University of New Jersey,

65 Dudley Road, New Brunswick, NJ 08901, USA

e-mail: tchikindas@aesop.rutgers.edu;

micromike123@yahoo.com

K. Sutyak Noll

Kraft Foods, Inc., 555 South Broadway, Tarrytown, NY 10591,

USA

\section{P. J. Sinko}

Department of Pharmaceutics, Ernest Mario School of

Pharmacy, Rutgers, The State University of New Jersey,

160 Frelinghuysen Road, Piscataway, NJ 08854, USA 